\title{
A flexible, multilayered protein scaffold maintains the slit in between glomerular podocytes
}

Florian Grahammer, ${ }^{1}$ Christoph Wigge, ${ }^{2}$ Christoph Schell, ${ }^{1,3,4}$ Oliver Kretz, ${ }^{1,5,6}$ Jaakko Patrakka, ${ }^{7}$ Simon Schneider, ${ }^{1}$ Martin Klose, ${ }^{8,9,10}$ Julia Kind, ${ }^{1}$ Sebastian J. Arnold,,${ }^{1,5,11}$ Anja Habermann, ${ }^{2}$ Ricarda Bräuniger, ${ }^{2}$ Markus M. Rinschen, ${ }^{12}$ Linus Völker, ${ }^{12}$ Andreas Bregenzer, ${ }^{1}$ Dennis Rubbenstroth, ${ }^{13}$ Melanie Boerries, ${ }^{8,9,10}$ Dontscho Kerjaschki, ${ }^{14}$ Jeffrey H. Miner, ${ }^{15}$ Gerd Walz, ${ }^{1}$ Thomas Benzing, ${ }^{12}$ Alessia Fornoni, ${ }^{16}$ Achilleas S. Frangakis, ${ }^{2}$ and Tobias B. Huber, ${ }^{1,3,5,17}$

'Department of Medicine, Division of Nephrology, Medical Center, Faculty of Medicine, University of Freiburg, Freiburg, Cermany. ${ }^{2}$ Buchmann Institute for Molecular Life Sciences and Institute for Biophysics, Goethe University Frankfurt, Frankfurt, Germany. ${ }^{3}$ Spemann Graduate School of Biology and Medicine, ${ }^{4}$ Faculty of Biology, and ${ }^{5}$ BIOSS Center for Biological Signalling Studies, Albert-Ludwigs University of Freiburg, Freiburg, Germany. ${ }^{6}$ Institute of Anatomy, Faculty of Medicine, University of Freiburg, Freiburg, Germany. ${ }^{7} \mathrm{KI} / \mathrm{AZ}$ Integrated Cardio-Metabolic Center (ICMC), Department of Laboratory Medicine, Karolinska Institutet at Karolinska University Hospital Huddinge, Stockholm, Sweden. ${ }^{8}$ Institute of Molecular Medicine and Cell Research, Faculty of Medicine, University of Freiburg, Freiburg, Germany. ${ }^{9}$ Cerman Cancer Consortium and ${ }^{10} \mathrm{German}$ Cancer Research Center, Heidelberg, Germany. ${ }^{11}$ Institute of Experimental and Clinical Pharmacology and Toxicology, Faculty of Medicine, University of Freiburg, Freiburg, Germany. ${ }^{12}$ Department II of Internal Medicine and Center for Molecular Medicine Cologne, University of Cologne, Cologne, Germany. ${ }^{13}$ Institute of Virology, Medical Center - University of Freiburg, Faculty of Medicine, University of Freiburg, Germany. ${ }^{14}$ Department of Pathology, University of Vienna, Vienna, Austria. ${ }^{15}$ Renal Division, Washington University, St. Louis, Missouri, USA. ${ }^{16}$ Division of Nephrology and Katz Family Drug Discovery Center, University of Miami Miller School of Medicine, Miami, Florida, USA. ${ }^{17}$ FRIAS, Freiburg Institute for Advanced Studies and ZBSA, Center for Biological System Analysis, Albert-LudwigsUniversity of Freiburg, Germany.

Authorship note: C. Wigge and C. Schell contributed equally to this work. A.S. Frangakis and T.B. Huber are co-senior authors.

Conflict of interest: The authors declare that no conflict of interest exists.

Submitted: December 28, 2015 Accepted: May 17, 2016

Published: June 16, 2016

Reference information: JCI Insight. 2016;1(9):e86177. doi:10.1172/jici.insight.86177.
Vertebrate life critically depends on renal filtration and excretion of low molecular weight waste products. This process is controlled by a specialized cell-cell contact between podocyte foot processes: the slit diaphragm (SD). Using a comprehensive set of targeted KO mice of key SD molecules, we provided genetic, functional, and high-resolution ultrastructural data highlighting a concept of a flexible, dynamic, and multilayered architecture of the SD. Our data indicate that the mammalian SD is composed of NEPHRIN and NEPH1 molecules, while NEPH2 and NEPH3 do not participate in podocyte intercellular junction formation. Unexpectedly, homo- and heteromeric NEPHRIN/NEPH1 complexes are rarely observed. Instead, single NEPH1 molecules appear to form the lower part of the junction close to the glomerular basement membrane with a width of $23 \mathrm{~nm}$, while single NEPHRIN molecules form an adjacent junction more apically with a width of $45 \mathrm{~nm}$. In both cases, the molecules are quasiperiodically spaced $7 \mathrm{~nm}$ apart. These structural findings, in combination with the flexibility inherent to the repetitive Ig folds of NEPHRIN and NEPH1, indicate that the SD likely represents a highly dynamic cell-cell contact that forms an adjustable, nonclogging barrier within the renal filtration apparatus.

\section{Introduction}

The identification of mutations in the type 1 transmembrane molecule NEPHRIN in hereditary nephrotic syndrome revealed its central role in kidney filtration barrier function (1). Subsequently, the NEPHRIN-related protein family of NEPH1-3 proteins was identified (2), and all 4 molecules have been implicated in the assembly of the slit diaphragm (SD). Their cytoplasmic tails appear to initiate crucial signalling programs that maintain the integrity of the SD (1,3-5), establishing the SD as a dynamic signalling hub that transfers and integrates diverse internal and external cues $(6,7)$. In addition to NEPHRIN and NEPH family proteins, molecular constituents of tight, adhesion, gap, and neuronal junctions localize to the $\mathrm{SD}$, 
establishing the SD as a unique cell-cell contact (7). Although our knowledge of the molecular composition of the SD complex has expanded over the past decade, the structure and structure-based functional concepts of the glomerular filtration barrier have remained incomplete. On the basis of conventional electron microscopy (EM) studies of perfusion-fixed rat kidneys, Rodewald and Karnovsky proposed a zipper-like SD structure (8). More recently, an electron tomography study of fixed tissue suggested that the SD contains 35-nm strands that wind together to form a network, creating pores smaller than albumin molecules (9). However, owing to the low accessibility of the SD in vivo and the absence of high-resolution studies on nonfixed tissue, the precise molecular structure and function of the SD remained unresolved. Moreover, several known properties of the glomerular filtration barrier are difficult to explain based on the current structural model, begging the following questions: how can an anticipated rigid mesh of NEPHRIN/ NEPH cis- and trans-heterodimers adapt to dynamic changes of podocyte foot processes; which roles are taken by the different members of the NEPH family proteins; and why does this static filter at the outermost section of the glomerular filtration barrier not clog? These unanswered questions led us to reappraise the molecular composition and ultrastructure of the slit diaphragm.

\section{Results}

Lack of NEPHRIN or NEPH1, but not NEPH2 or NEPH3, causes early lethality and severe nephrotic kidney disease. Since the reported expression profiles of all 4 NEPHRIN/NEPH protein family members varied considerably throughout the literature, we first performed ISH to determine their individual expression profiles $(3,4,10)$. Tissue from newly generated constitutive KO mice for Nphs1, Neph1, Neph2, and Neph3 was used as a negative control. Nphs1 (NEPHRIN) showed a restricted expression pattern in podocytes, pancreas, and the cerebellum anlage (Figure 1, A and B and Supplemental Figure 1, A-D; supplemental material available online with this article; doi:10.1172/jci.insight.86177DS1). In contrast, ISH and Western blotting revealed that Neph1 was broadly expressed in kidney, lung, brain, heart, skeletal muscle, liver, pancreas, and gut (Figure 1, C and D and Supplemental Figure 1, E-I). Neph2 was expressed predominantly in the central nervous system and peripheral neuronal tissue, whereas Neph3 was expressed mainly in the hind- and forebrain, as well as the pancreas (Supplemental Figure 2, A and B) (11). Surprisingly, despite their proposed function within the SD, neither Neph2 nor Neph3 were detected in embryonic kidney by ISH (Supplemental Figure 2, A and B) $(4,5,12)$. Adult expression in 8-week-old animals was assessed using isolated podocytes of $m T / m G^{*} h N P H S 2 C r e$ mice. While Nphs1 and Neph1 mRNA could be readily detected, no Neph2 mRNA and only a weak band corresponding to Neph3 mRNA could be demonstrated (Supplemental Figure 2E). In contrast to adult brain tissue, no NEPH2 and NEPH3 protein could be detected in glomerular lysates (Supplemental Figure 2F)

To further examine the functional role of NEPHRIN, NEPH1, NEPH2, and NEPH3, we generated constitutive KO mice for each gene (Figure 1, E-H; Supplemental Figure 1, J-M; and Supplemental Figure 2, C and D). These mice displayed differing survival characteristics; $N p h s 1^{-/-}$and $N e p h 1^{-/-}$mice were born with nephrotic-range proteinuria and died perinatally, whereas $N e p h 2^{-/-}$and $N e p h 3^{-/-}$mice appeared normal for at least the first 6 months (Figure 1, E-H).

NEPHRIN and NEPH1 can form rudimentary SDs independently of each other. By light microscopy, both $N p h s 1^{-/-}$and $\mathrm{Neph}^{-/-}$had glomerula of normal appearance but presented signs of increased tubular protein reuptake (Figure 2, A, E, and I). Compared with control animals, scanning EM (SEM) revealed broadened primary processes, as well as shortened foot processes in $\mathrm{Nphs}^{-1^{--}}$and $\mathrm{Neph1^{-/- }}$ mice (Figure 2. B, F, and J). Transmission EM (TEM) documented tight junction-based intercellular contacts in most areas of podocytes covering the glomerular basement membrane (GBM) (Figure 2, C, G, and K). NEPH1 immunoreactivity in $\mathrm{Nphs}^{1^{-/-}}$kidneys was largely maintained, as was NEPHRIN expression in $N e p h 1^{-1-}$ (Figure 2, D, H, and L). Unexpectedly, in 17\% of the GBM, we detected shorter $22 \mathrm{~nm}$ intercellular junctions in $\mathrm{Nphs}^{-1-}$ mice (Figure 2, M and $\mathrm{N}$ and Supplemental Figure 3, A and C), which previously have not been described (13).

Importantly and in contrast to the current literature, this kind of intercellular junction was also seen in TEM images of human patients with congenital nephrotic syndrome (CNS) due to NPHS1 Fin major/Fin major mutations (Figure 2S, Supplemental Figure 3C, and Supplemental Figure 4, A-E) (14-17). Immunogold labeling identified these structures as NEPH1-containing cell-cell contacts in both mice and humans

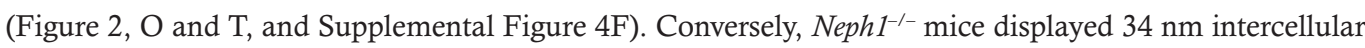
junctions in $30 \%$ of the GBM at early stages of glomerular maturation (Figure 2, P and Q and Supplemen- 

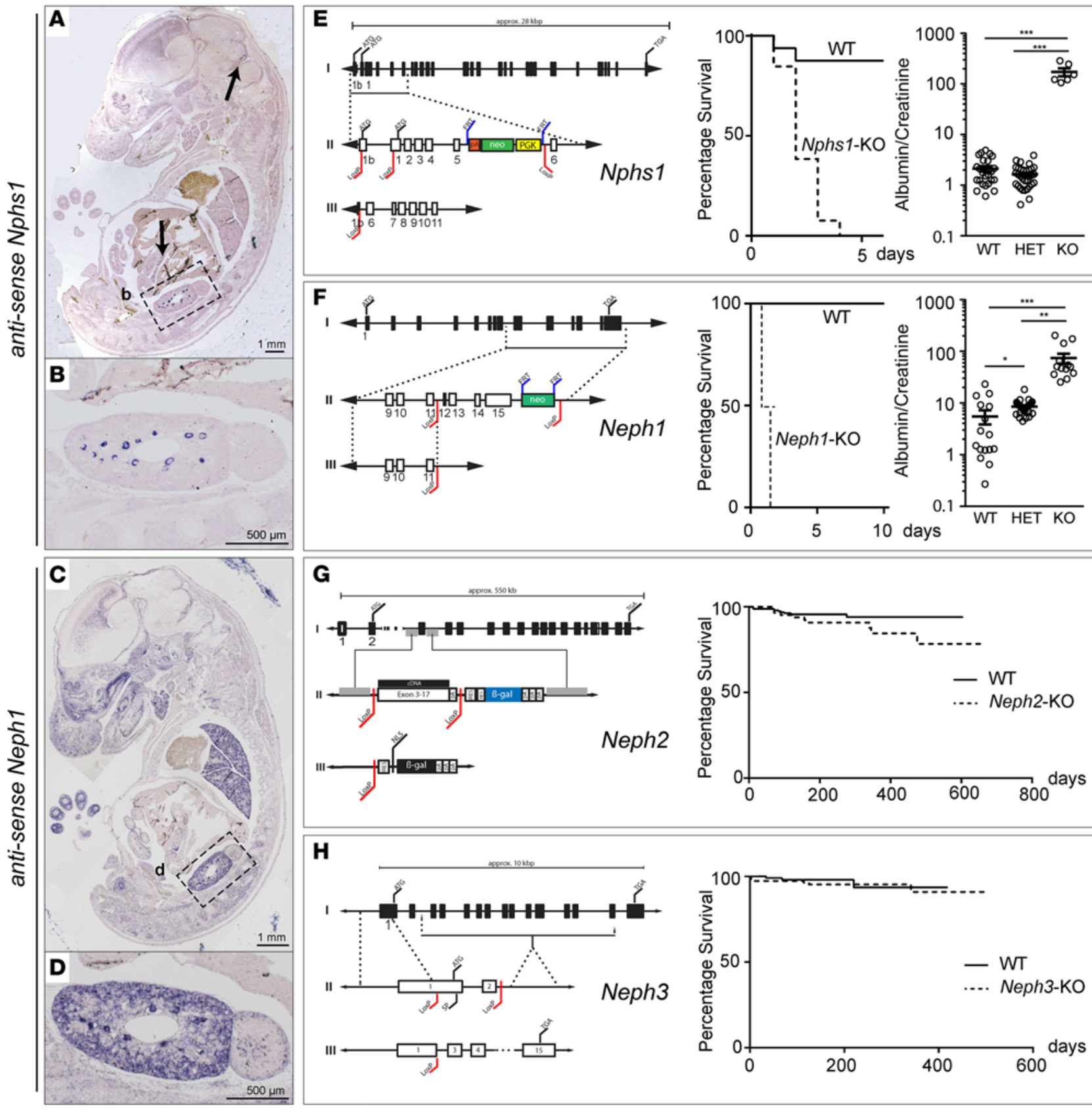

Figure 1. Lack of NEPHRIN or NEPH1, but not NEPH2 or NEPH3, causes early lethality and severe nephrotic kidney disease. (A and B) At E13.5, Nphs1 showed a restricted expression pattern in renal glomeruli, pancreas, and cerebellum anlage. First arrow, cerebellum anlage; second arrow, pancreas; box labeled $b$ is the magnified area shown in B. (C and D) At E13.5, Neph1 was broadly expressed in the central nervous system, lung, kidney, and gut. Box labeled d is the magnified area shown in D. (E-H) Targeting strategy and survival analysis of constitutive Nphs1, Neph1, Neph2, and Neph3 KO mice. Constitutive KO of Nphs1 or Neph1 led to early perinatal lethality, whereas mice deficient in Neph2 or Neph3 did not die prematurely (log rank [Mantel-Cox] test was used with at least 10 animals per

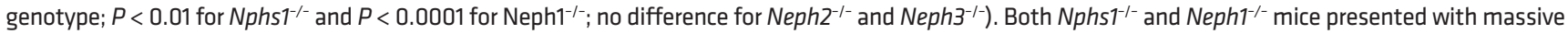
proteinuria (at least 5 animals per genotype and allele were analyzed for urinary albumin and creatinine; ${ }^{*} P<0.05,{ }^{*} P<0.01,{ }^{* * *} P<0.001$ ).

tal Figure 3, B and C). During maturation, these remnant SDs moved apically and were finally replaced by apparent tight junctions (Figure $2 \mathrm{~K}$ and Supplemental Figure 3, D-F). Immunogold labeling of these early SD-like junctions identified NEPHRIN as a constituent (Figure 2R). Loss of either Nphs1 or Neph1 led to pronounced proteinuria at birth, whereas Neph2 and Neph3 KO mice had normal-appearing SDs and did not show any signs of proteinuria (Supplemental Figure 5, A-H). Together, these data suggest that the mammalian SD consists of 2 main functional components, a NEPH1- and a NEPHRIN-based cell-cell contact, both of which are required for normal SD development and maintenance in mammals. 

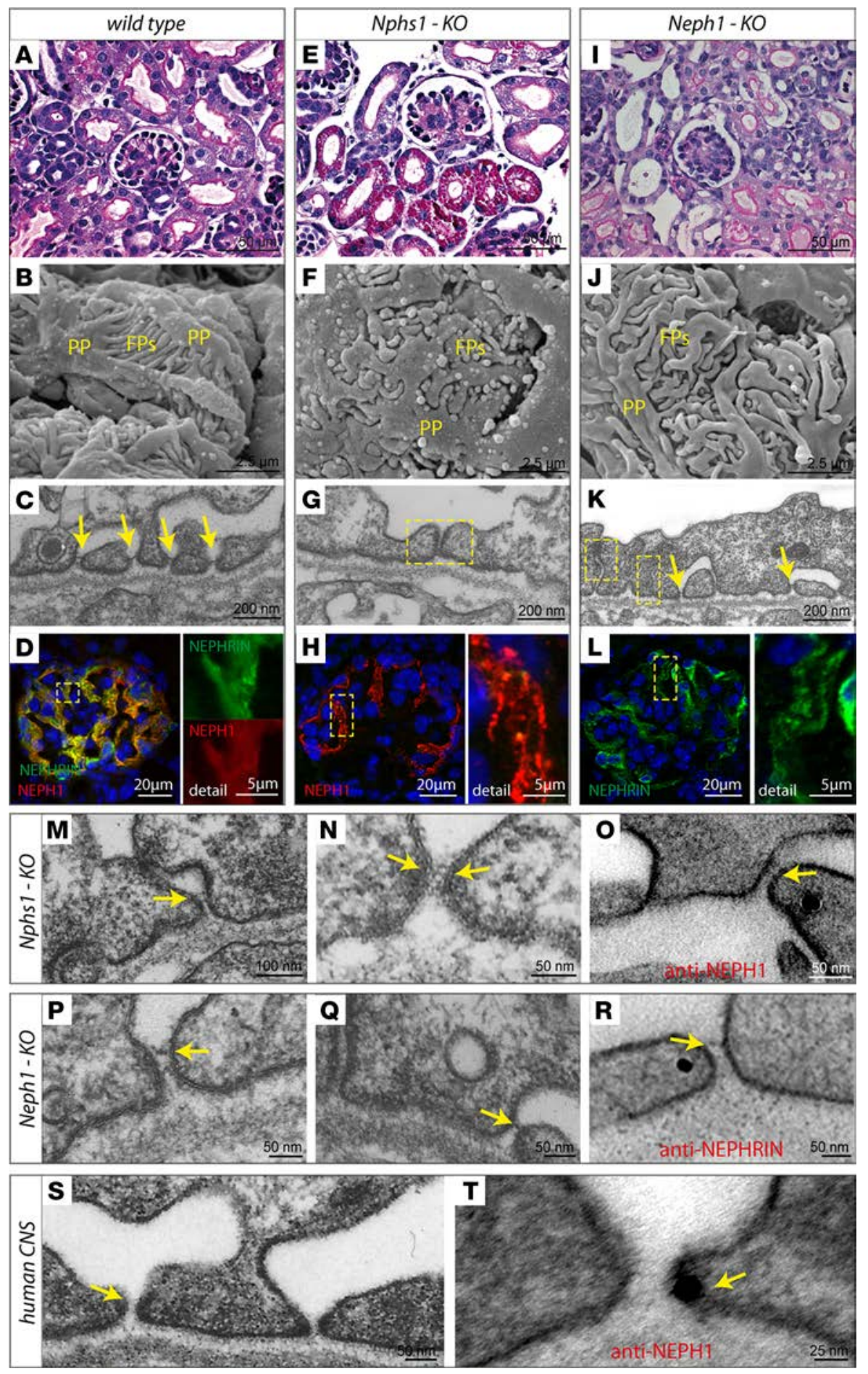

Figure 2. NEPHRIN and NEPH1 can form rudimentary SDs independently of each other. (A-C and E-G) In contrast to control mice, Nphs ${ }^{-1}$ mice had protein-filled proximal tubular cells, broadened primary (PP) and shortened secondary foot processes (FP), and what appeared to be tight junctional cell-cell contacts between effaced foot processes. Yellow arrows represent SDs slit diaphragms; yellow squares represent tight junctions. (I-K) Neph1-1- mice appeared to have misdirected foot processes but, at least at early stages of glomerular maturation, were able to form SD-like junctions. (D, H, and $\mathbf{L}$ ) Immunoreactivity for NEPH1 was largely maintained in $\mathrm{Nphs}^{-/-}$, as was staining intensity for NEPHRIN in $\mathrm{Neph1}^{-1-}$. (M-O) Further evaluation of Nphs1-deficient glomeruli demonstrated thin and short strand-like junctions between incompletely effaced foot processes that showed NEPH1 immunoreactivity. (P-R) Similarly, in $\mathrm{Neph}^{-/-}$mice, we detected SD-like junctions that often appeared dislocated apically and had NEPHRIN immunoreactivity. ( $\mathbf{S}$ and $\mathbf{T}$ ) In tissue from human CNS patients with remaining open slits, SD-like junctions could be visualized, showing immunoreactivity for NEPH1.

The $S D$ is a flexible multilayered, bipartite protein scaffold. To analyze the precise arrangement of NEPHRIN and NEPH1 within the mammalian SD, we used cryo-electron tomography (CET) of vitreous sections (Figure 3, A and B) (18). The tomograms revealed quasiperiodic molecular strands, spanning adjacent podocyte foot processes. These strands typically formed 2-3 individual layers toward the apical cell surface (Figure 3A). Although the length of these molecular strands varied markedly between the basal and apical layers, they exhibited a similar thickness and approximately the same distance to the neighboring molecule, indicating that homophilic interactions in a trans-configuration were virtually absent (Figure 3, $\mathrm{A}$ and $\mathrm{B}$ ). The length of 283 individual molecular strands extracted from individual subtomograms varied between 15 and 55 nm. Subsequent subtomogram averaging and classification of the strands according to their length revealed a quasiperiodic spacing of $7 \mathrm{~nm}$, which becomes more disordered with increasing length. The strand length, as quantified after classification, shows a bimodal distribution with 2 peaks at $25 \mathrm{~nm}$ and $40 \mathrm{~nm}$, respectively.

Homology modeling of NEPHRIN and NEPH1 with TITIN and other Ig-fold containing proteins (sequence identities between $39 \%-78 \%$, Supplemental Table 1) suggested that the extracellular domain of NEPHRIN contains $9 \mathrm{Ig}$ folds and measures $43.8 \mathrm{~nm}$ in length, while the extracellular domain of NEPH1 contains $5 \mathrm{Ig}$ folds, resulting in a length of $19.7 \mathrm{~nm}$ (Figure 4, B and C and Supplemental Table 1) (19, 20). These values are consistent with the measured strand lengths of the SD in vitreous sections (Figure 3, A and B). Due to large variations of individual strand lengths, subtomogram averaging did not yield a high-resolution 

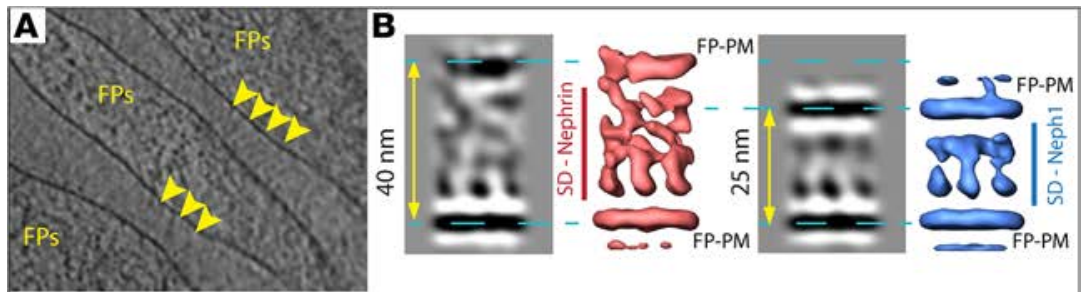

transversal - view
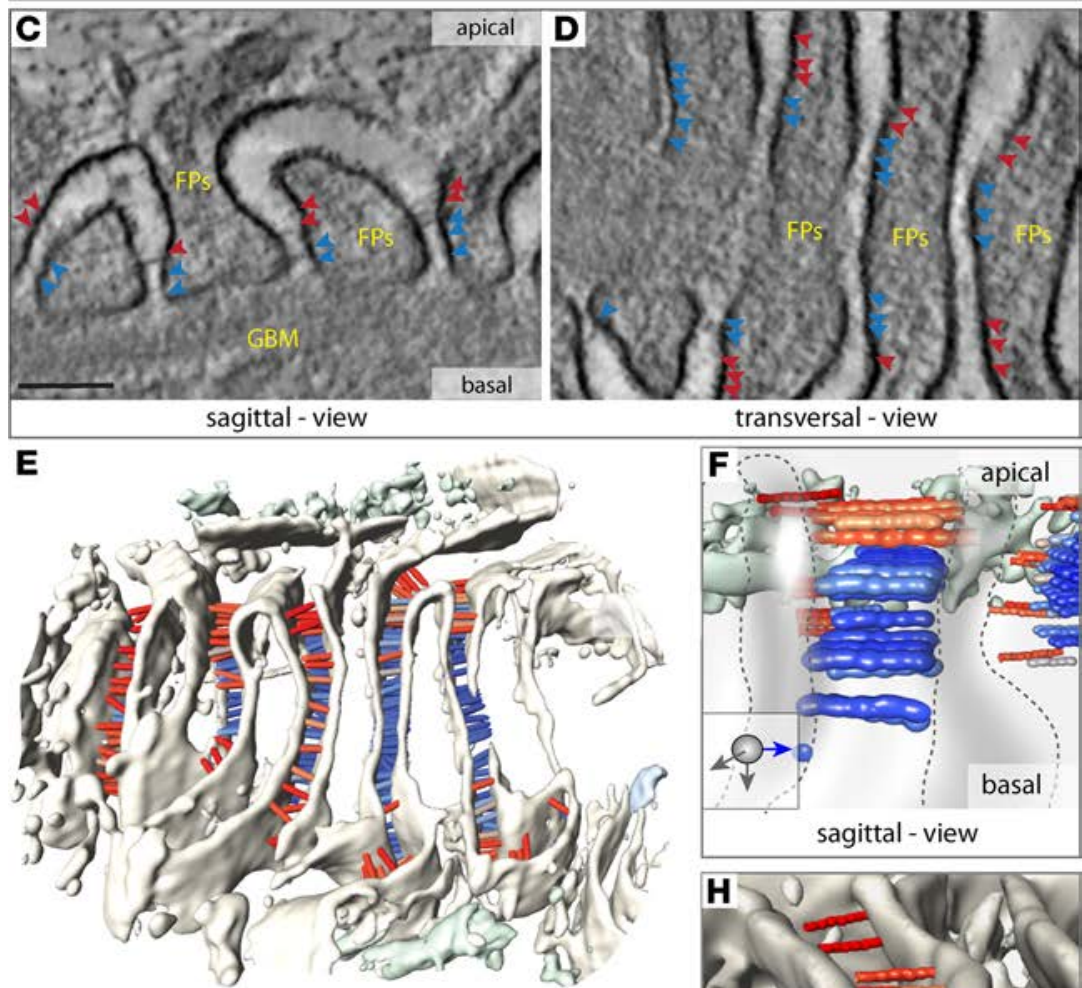

Rendered SD junctions with distance color code

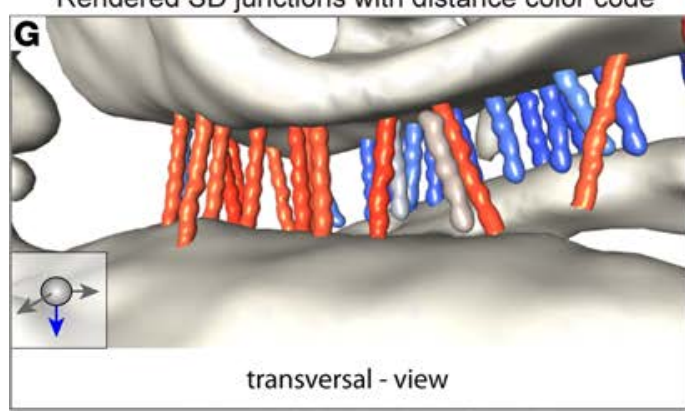

transversal - view
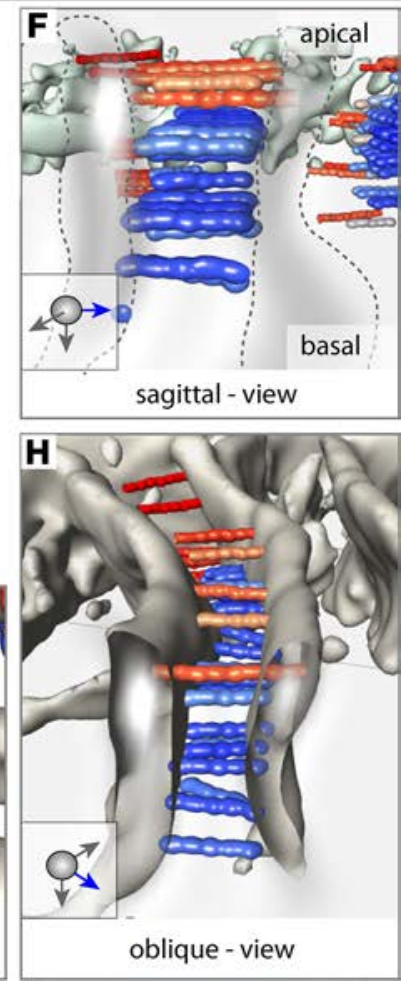

Figure 3. The SD is a multilayered, bipartite protein scaffold. (A) CET (cryo-electrontomography) of vitreous sections: Tangential computational 2-nm thick section through the base of several foot processes (FP). Individual densities (indicated by the yellow arrowheads) can be seen spanning the area between 2 FP. They are quasiperiodically arranged and have similar thickness, without a dense midline. (B) Subtomogram averaging of these densities reveals 2 predominant classes; the first class has a intermembrane distance of $40 \mathrm{~nm}$ (corresponding isosurface representation shown in red). The second has an intermembrane distance of $25 \mathrm{~nm}$ (isosurface representation shown in blue). For both entities, quasiperiodic strands with a thickness of $3 \mathrm{~nm}$ can be seen spanning the membranes. With increasing distance, the strands appear more disordered, while the shorter ones are rather regularly arranged. A dense midline cannot be discerned. (C) Computational sagittal section (10-nm thick) from a PET tomogram showing the typical appearance of podocytes. Several strands cross the individual FPs with the narrower end of the slit toward the GBM, while the broader opening faces Bowman's space. The colored arrowheads point to individual strands. Red arrowheads correspond to longer strands, while blue arrowheads mark shorter strands. (D) Transversal computational section (10-nm thick) (in a similar orientation as $\mathbf{A}$ ) showing the quasiperiodic arrangement of several strands (color code as in $\mathbf{C}$ ). (E) Surface visualization (3D) seen from Bowman's capsule showing several FP and hundreds of bridging strands color-coded depending on their length (red $40 \mathrm{~nm}$, blue $25 \mathrm{~nm}$; color variation indicating deviations from these). The histogram of their length variation is shown in Figure 4A. (F) Typical sagittal view of 2 FP, showing 3 layers of 25-nm strands, which we attribute to NEPH1 (blue, and $5 \mathrm{Ig}$ repeats) and one layer of $40-\mathrm{nm}$ strands that we attribute to NEPHRIN (red, and 9 Ig repeats). (G) Transversal view showing a quasiperiodic arrangement of NEPHRIN molecules. (H) Oblique view from Bowman's capsule onto the molecular arrangement of NEPHRIN and NEPH1.

structure. Thus, we used high-pressure freezing, freeze substitution, and plastic electron tomography (PET) of the same specimen to record larger fields of view and to obtain reliable statistics by measuring the length of several thousand individual molecular strands. PET confirmed the tight quasiperiodic arrangement of NEPH1 molecules in a junctional compartment facing the GBM (Figure 3, C-H), while NEPHRIN strands in the junctional compartment distant to the GBM were loosely stacked and less periodically arranged (Figure $3, \mathrm{E}-\mathrm{H})$. Analyzing the length of strands connecting the interdigitating podocyte foot processes revealed a bimodal peak at $23 \mathrm{~nm}$ and $45 \mathrm{~nm}$ (with a variance of $10 \mathrm{~nm}$ ), corresponding well to the CET data analysis (Figure 4A). The PET 3D reconstruction showed a multilayered, bipartite assembly of the SD with several horizontal layers of basal NEPH1 strands and apical NEPHRIN strands crossing the interpodocyte space (Figure 3, E-H). The 3D reconstruction suggests an approximately 1:2.5 ratio of NEPHRIN and NEPH1 molecules within the SD. Interestingly, we could obtain this molecular ratio reanalyzing available mouse and human proteomic data sets, which — in the case of microdissected human glomerula — yielded a ratio of 
A

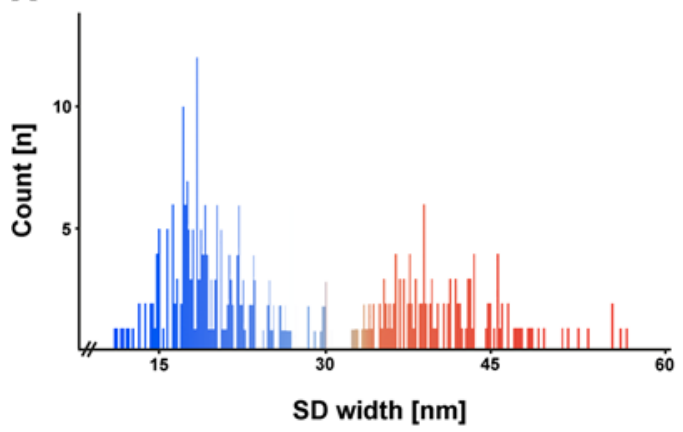

B

Nphs1

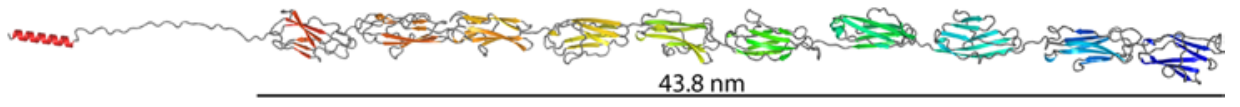

C

Neph1

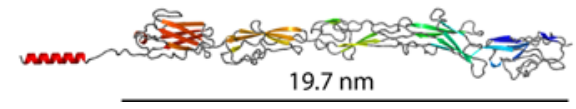

Figure 4. Structural modeling of NPHS1 and NEPH1 based on their homology to TITIN. (A) Length distribution of individual strands within mouse foot processes in PET reconstructions gives 2 gaussian-like distributions centered at $45 \mathrm{~nm}$ and $23 \mathrm{~nm}$. (B) For mouse NPHS1, databases (uniprot: Q9QZS7, ncbi: NP_062332.2) predict $8 \mathrm{~N}$-terminal immunoglobulin type C2 domains and 1 C-terminal fibronectin type III domain. A ninth Ig domain (between Ig6 and Ig7, aa 650-753) was predicted with sequence alignments of all mouse NPHS1 lg domains and with the use of PHYRE2 (36). Straight length for NEPHRIN was estimated at $43.8 \mathrm{~nm}$. (C) In accordance with the literature, mouse NEPH1 (uniprot Q80W68, ncbi: NM_019459.2) consists of $5 \mathrm{~N}$-terminal immunoglobulin type C2 domains. In the case of NEPH1, straight length was estimated to be $19.7 \mathrm{~nm}$.

1:2.34 for the amount of NEPHRIN to NEPH1 protein $(\operatorname{SEM} \pm 0.53, n=7)(21,22)$.

Evolutionary concept of a NEPH1-based SD in birds. Given the complexity of the multilayered, bipartite (NEPHRIN/NEPH1) mammalian SD, we investigated the evolutionary conservation of the genes encoding for NEPHRIN and NEPH1 (www.treefam.org, TF327139). Whereas NEPH1 is remarkable conserved through all 99 species examined, NPHS1 encoding for NEPHRIN is surprisingly absent in birds (Figure 5A). Strikingly, however, we found that birds (chicken; Gallus gallus), despite the absence of NEPHRIN, still exhibited a fully differentiated foot process network with SDs (Figure 5, B and C, E-G). Thus, this animal model offered the unique opportunity to explore the junctional architecture of a non-NEPHRIN based SD. Supporting our hypothesis that the shorter SDs in mammals are formed by NEPH1, the SD in G. gallus had a width of $18 \mathrm{~nm}$, similar to the $22 \mathrm{~nm}$-wide SD in $\mathrm{Nphs}^{1^{--}}$mice or the $23 \mathrm{~nm}$-wide SD in humans with CNS due to NPHS1 mutations (Figure 5D). As predicted by the genome analysis, we detected NEPH1, but not NEPHRIN, by immunofluorescence and immuno-electron microscopy in chicken kidneys (Figure 5I and Supplemental Figure 6, A-F). Other known SD-associated proteins (e.g., PODOCIN, ZO-1, CD2AP, and TRPC6) were detected in G. gallus, indicating an otherwise similar molecular composition of this structure (Figure 5, H, and J-L and Supplemental Figure 6, G-L, refs. 23-27). High-resolution SEM analysis of the $\mathrm{SD}$ in $G$. gallus revealed a tight mesh of junctions spanning the interpodocyte space similar to the NEPH1 molecule configuration in the mammalian SD (Figure 5, O and P). This is in contrast to mice, in which multiple molecular strands that we attribute to NEPHRIN bridge the interpodocyte space (Figure 5, M and N). Importantly, birds have a more than $20 \times$ higher urinary protein concentration $(2.0 \mathrm{mg} / \mathrm{ml} \mathrm{vs} .<0.1 \mathrm{mg} / \mathrm{ml})$ than mammals - a difference that appears to be needed to keep uric acid in a micellar solution. Thus, our data might suggest that the efficient excretion of nitrogen via uric acid in a high-protein containing solution is associated with the evolutionary loss of NEPHRIN in birds.

\section{Discussion}

In recent years, the SD has emerged as a key substructure in glomerular disease and proteinuria. With the cloning of Nphs1 as the gene responsible for CNS of the Finnish type has allowed analysis of this 
A
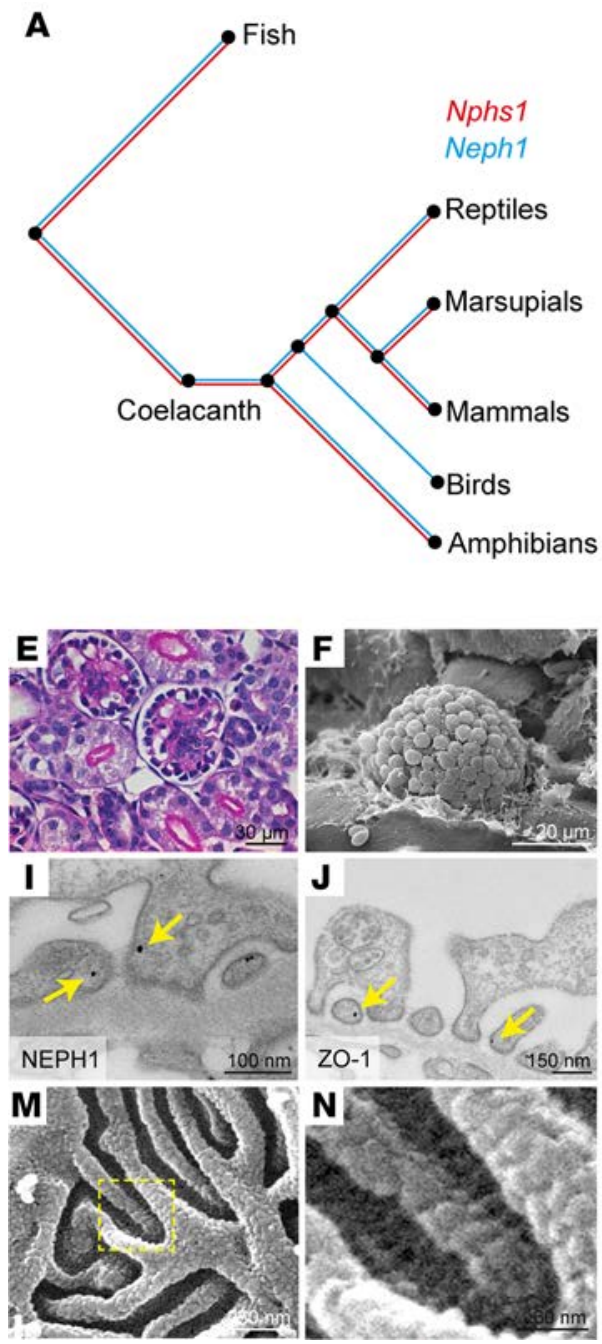
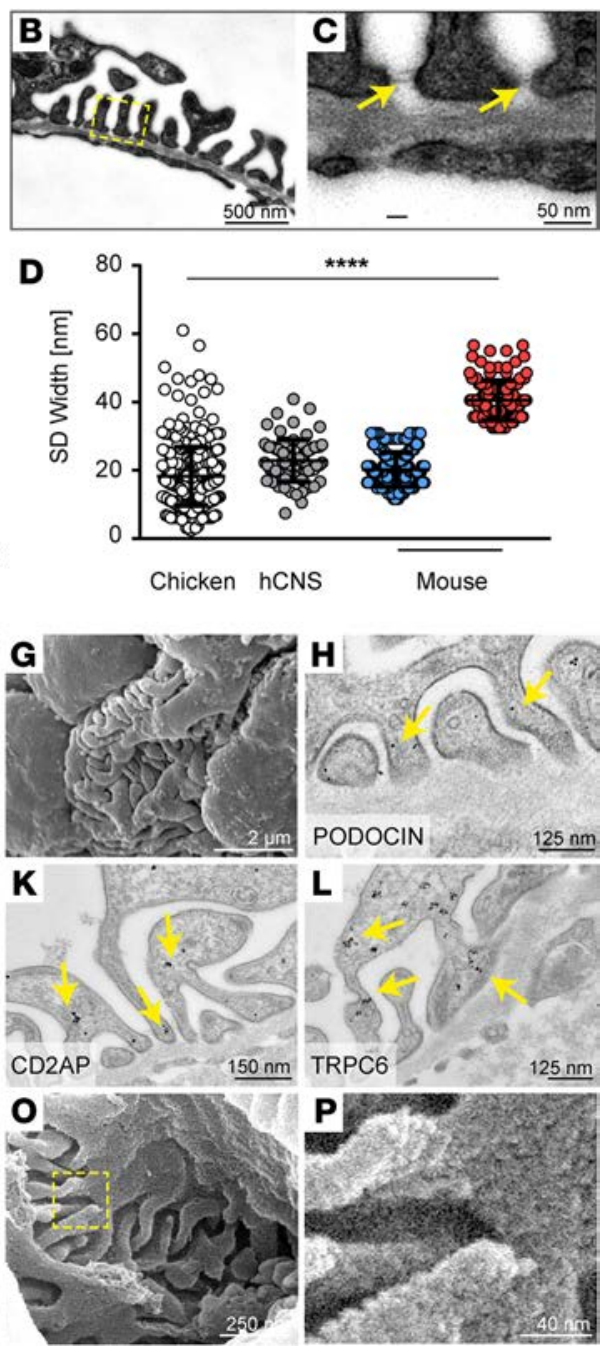

Figure 5. Evolutionary concept of a NEPH1-based SD formation in birds. (A) Phylogenetic analysis of Nphs1 and Neph1 throughout the animal kingdom. (B and C) Coelacanths contain both genes, whereas Nphs1 was lost only in birds. TEM reveals that foot processes are evenly spaced, and multiple layers of slit diaphragms can be detected in Gallus gallus. (D) Analysis of SD width illustrates that the chicken SD is similar to the one found in human CNS (hCNS), while mice have an SD containing both narrow and wide cell-cell junctions (chicken, $n=391$; human, $n=77$; mouse NEPH1, $n=360$; NEPHRIN, $n=77$; all $n$ quantities refer to individual measurements in at least $n=3$ different biological samples). ${ }^{* * *} P$ $<0.0001$ by ANOVA. (E-G) Light microscopy images demonstrate that podocyte cell bodies in G. gallus are found more toward the edge of the glomerular field directly opposite Bowman's capsule, whereas SEM reveals broadened primary and shortened secondary foot processes compared with mice. (H-L) Established SD molecules - including PODOCIN, NEPH1, ZO-1, CD2AP, and TRPC6 - can be detected at the chicken SD. Arrows indicate immunogold particles. ( $\mathbf{M}$ and $\mathbf{N}$ ) High-resolution SEM of mouse glomeruli reveals individual strands of molecules crossing the slit above an interwoven molecular mesh situated in the lower part of this intercellular junction. ( $\mathbf{O}$ and $\mathbf{P})$ Chicken glomeruli contain the mesh but lack the individual strands. Boxes in $\mathbf{M}$ and $\mathbf{O}$ indicate magnified area shown in $\mathbf{N}$ and $\mathbf{P}$, respectively.

condition at the molecular level (1). Further understanding of the role of NPHS1 in the development of CNS has also been fostered by the use of $\mathrm{KO}$ animals targeting newly identified constituents of the SD. While, for all 3 previous $N$ phs 1 KO models $(13,28,29)$, perinatal lethality and extreme albuminuria similar to our mouse model were reported, description of a substantial percentage of open slits $(20 \%)$ showing some residual parts of a shortened slit diaphragm $(22 \mathrm{~nm})$ is a new observation that is in contrast to the so far noted complete loss of the SD. Given that this finding could be robustly shown in both mouse and human tissue, differences in either mouse genetic background or tissue preparation might be a possible explanation for the observed differences.

While others have shown RNA and protein expression of all $3 \mathrm{NEPH}$ family members in glomerula, 
we - in contrast - can demonstrate that, beside Neph1, only Neph3 is expressed at the level of RNA in podocytes, while on a glomerular protein level, neither NEPH2 nor NEPH3 are detectable. This is underlined by the absence of a proteinuric phenotype in constitutive Neph2 and Neph3 KO animals $(4,30)$. It should be stated that both antibodies used were controlled using brain lysates of the respective constitutive $\mathrm{KO}$ animals. This evidence on the protein level, plus the lack of a functional glomerular phenotype in both Neph 2 and Neph $3 \mathrm{KO}$ animals, in our opinion conclusively indicates that NEPH1 is the only relevant player of the NEPH family of proteins at the SD.

Although the composition and function of the SD in part has been described before, the native molecular architecture and function of its constituents have remained elusive. Electron tomography revealed 2 populations of strands: one with an average length of 23 (variance $10 \mathrm{~nm}$ ) and another with an average length of $45 \mathrm{~nm}$ (variance $10 \mathrm{~nm}$ ). The extreme variability seen in individual strand lengths is in striking contrast to the minimal flexibility usually observed in cadherin-based junctions, such as desmosomes or adherens junctions (18). The measurements of the inter-foot process distances in capillaries without blood flow correspond well to the extracellular lengths of both NEPH1 and NEPHRIN at a low energy state. Bioinformatic analysis revealed a high homology of NEPH-1 and NEPHRIN to TITIN, OBSCURIN-like proteins, VCAM-1, DSCAM, and other immunoglobulin superfamily molecules; several of these molecules have been shown to function as molecular springs (31), a mechanism utilized within biological systems to provide a high degree of flexibility. Among these, TITIN is probably the best-studied protein and functions as a return spring to reposition the muscular thick filaments (32). Atomic force microscopy and mechanotransduction experiments have shown that the TITIN Ig-fold repeats can be dramatically stretched. A possible molecular spring mechanism of NEPH1 and NEPHRIN could be supported by the extreme variability seen in individual strand lengths. Conceptually, a spring-like, bipartite arrangement of NEPH1 and NEPHRIN could account for the dynamic adjustments of the SD required to respond to different blood pressures and variable glomerular capillary wall extensions and could offer an evolving concept of the biophysical properties and function of the SD. Firstly, the peculiar hourglass shape of adjacent podocyte foot processes can be explained by the layered, bipartite arrangement of NEPH1 and NEPHRIN. The shorter NEPH1 molecules bridge the narrower basal parts of the intercellular slit, providing a broad contact area with the GBM and concentrating the adhesive properties of the foot processes on the side opposing the GBM. The longer NEPHRIN molecules are placed more apically and follow the shape of the foot process. Secondly, the molecular spring properties of NEPH1 and NEPHRIN may have extensive implications for the filtration properties of podocytes. Our tomographic reconstructions revealed that transinteractions near the SD midline are very rare $(<5 \%)$, and thus, the previously postulated dense midline was virtually absent. Historically, this dense midline structure was based on conventional EM techniques and laid the foundation of the molecular zipper SD concept and of a static sieve model, blocking molecules larger than the size of albumin $(8,9)$. Based on our observations, the structure of NEPH1 and NEPHRIN suggest the possibility of a more dynamic arrangement (20). In the relaxed state, their layered assembly could allow the diffusion of small molecules approximately $2-3 \mathrm{~nm}$ in diameter. Changing the slit width (by e.g. blood pressure variation, glomerular contractions, body movements) could lead to elongation or shortening of the molecular springs formed by NEPH1 and NEPHRIN. During these repetitive cycles of strain and relaxation, NEPH1 and NEPHRIN would actively change their spacing, while adaptive changes in NEPH1/NEPHRIN-mediated signaling and rearrangement of the cortical actin web could control the slit width. This would allow molecules like albumin to move through the SD at presumably low rates, which ultimately could ensure that the filter does not clog.

Recently, two other groups reported results using block-face SEM (FIB-SEM) where they could resolve the arrangement of neighboring podocyte foot processes and prove that individual podocytes indeed only interdigitate with adjacent podocytes but not with their own processes $(33,34)$. However, this technique does not have the resolution to study the SD in detail. One of these groups, therefore, also used electron tomography, albeit with glutaraldehyde fixed samples (34). This might be the reason this group only found one continuous layer of cell-cell contacts. Whether the also-reported punctated filamentous cell-cell contacts on both sides of this SD monolayer represent remnants of the native structure, as we describe it in this manuscript, remains to be shown.

Our study also reveals that NEPHRIN is not required to build a functional filtration barrier in birds. While other IgG molecules such as adherens junction proteins have been shown in avian podocytes using 
antibody-based staining techniques, their relevance for maintaining the avian SD need to be determined (35). Interestingly, the bipartite NEPHRIN/NEPH1 organization seems to provide the filtering characteristics with the least loss of albumin. In birds, an SD containing only Neph1 has possibly evolved to keep urate in micellar solution, albeit at the expense of a higher proteinuria. The concept of a spring-like, multilayered interpodocyte slit stresses the necessity for a strong yet flexible cell-cell contact that forms an adjustable barrier to proteins, thereby ensuring that the renal filter does not clog at its outermost part.

\section{Methods}

Animals. Mice were housed in an specific pathogen free (SPF) facility with free access to chow and water and a 12-hour day/night cycle. Breeding and genotyping were done according to standard procedures. Urinary albumin and creatinine were measured using a fluorimetric albumin test kit (Progen, PR2005) or enzymatic colorimetric creatinine kit (LT-SYS, Lehmann) following the manufacturers' instructions.

For complete information on techniques used, please refer to the Supplemental Methods.

Statistics. Data are expressed as mean \pm SEM. Statistical comparisons were performed using the GraphPad Prism Software Package 6.02 (GraphPad Software) with 2-tailed Student's $t$ test, Wilcoxon test, or ANOVA, including respective corrections where indicated. Differences with $P$ values less than 0.05 were considered significant.

Study approval. All animal experiments were conducted according to the NIH Guide for the Care and Use of Laboratory Animals, as well as in accordance with the German law for the welfare of animals, and were approved by local authorities (Regierungspräsidium Freiburg G-09/23, G-10/100, X12/06J, and X13/04J).

\section{Author contributions}

FG, ASF, JM, and TBH conceived and analyzed experiments. FG, ASF, and TBH supervised the study. FG, CW, CS, OK, JP, SS, MK, SA, AH, RB, MMR, LV, AB, DR, MB, DK, and ASF performed experiments. GW, TB, and AF provided critical reagents. FG, CS, GW, ASF, and TBH wrote the manuscript with input and discussion from all authors.

\section{Acknowledgments}

We would like to thank Charlotte Meyer, Temel Kilic, Helga Schachner, and Elisabeth Wiesner for expert technical assistance. We thank Hannu Jalanko for granting us access to human EM material and Yutaka Yoshida for access to his original proteomic data sets of human glomeruli. In addition, we would like to express our gratitude to all members of our laboratories for helpful discussions and support. We thank Ketan Patel for critical reading of the manuscript. This study was supported by the German Research Foundation (DFG), CRC 1140 (to F. Grahammer, G. Walz, and T.B. Huber), CRC 992 (to T.B. Huber), Heisenberg program (to T.B. Huber), and FR 1653/3-1/HU 1016/5-1; the European Research Council (ERC grant to A.S. Frangakis and T.B. Huber); the BMBF-Joint transnational Grant 01KU1215 (T.B. Huber) and STOP-FSGS 01GM1518C (to T.B. Huber); the Else-Kröner Fresenius Stiftung-NAKSYS; Excellence Initiative of the German Federal and State Governments (GSC-4, Spemann Graduate School to C. Schell and T.B. Huber, BIOSS to T.B. Huber); the Diabetes Research Institute Foundation (A. Fornoni); and the NIH (R01-DK090316 and R01-DK104753 to A. Fornoni).

Address correspondence to: Achilleas S. Frangakis, Cluster of Excellence Macromolecular Complexes, Johann Wolfgang Goethe University Frankfurt, Institute of Biophysics, Max-von-Laue-Str. 15, D-60438 Frankfurt am Main, Germany. Phone: 49.69.79846462; Fax: 49.69.79846467; E-mail: achilleas. frangakis@biophysik.org. Or to: Tobias B. Huber, Uniklinikum Freiburg, Zentrale Klinische Forschung, Breisacherstrasse 66,79106 Freiburg, Germany. Phone: 49.761.27035590; Fax: 49.761.27063930; E-mail: tobias.huber@uniklinik-freiburg.de.

1. Kestila M, et al. Positionally cloned gene for a novel glomerular protein--nephrin--is mutated in congenital nephrotic syndrome. Molecular Cell. 1998;1(4):575-582.

2. Sellin L, Huber TB, Gerke P, Quack I, Pavenstädt H, Walz G. NEPH1 defines a novel family of podocin interacting proteins. FASEB J. 2003;17(1):115-117.

3. Donoviel DB, et al. Proteinuria and perinatal lethality in mice lacking NEPH1, a novel protein with homology to NEPHRIN Mol Cell Biol. 2001;21(14):4829-4836. 
4. Gerke P, et al. NEPH2 is located at the glomerular slit diaphragm, interacts with nephrin and is cleaved from podocytes by metalloproteinases. J Am Soc Nephrol. 2005;16(6):1693-1702.

5. Ihalmo P, Palmén T, Ahola H, Valtonen E, Holthöfer H. Filtrin is a novel member of nephrin-like proteins. Biochem Biophys Res Commun. 2003;300(2):364-370.

6. Huber TB, Benzing T. The slit diaphragm: a signaling platform to regulate podocyte function. Curr Opin Nephrol Hypertens. 2005;14(3):211-216

7. Grahammer F, Schell C, Huber TB. The podocyte slit diaphragm--from a thin grey line to a complex signalling hub. Nature Reviews Nephrology. 2013;9(10):587-598.

8. Rodewald R, Karnovsky MJ. Porous substructure of the glomerular slit diaphragm in the rat and mouse. J Cell Biol. 1974;60(2):423-433.

9. Wartiovaara J, et al. Nephrin strands contribute to a porous slit diaphragm scaffold as revealed by electron tomography. J Clin Invest. 2004;114(10):1475-1483.

10. Putaala H, Sainio K, Sariola H, Tryggvason K. Primary structure of mouse and rat nephrin cDNA and structure and expression of the mouse gene. J Am Soc Nephrol. 2000;11(6):991-1001.

11. Völker LA, et al. Comparative analysis of Neph gene expression in mouse and chicken development. Histochem Cell Biol. 2012;137(3):355-366.

12. Sun C, et al. Kirrel2, a novel immunoglobulin superfamily gene expressed primarily in beta cells of the pancreatic islets. Genomics. 2003;82(2):130-142.

13. Putaala H, Soininen R, Kilpeläinen P, Wartiovaara J, Tryggvason $\mathrm{K}$. The murine nephrin gene is specifically expressed in kidney, brain and pancreas: inactivation of the gene leads to massive proteinuria and neonatal death. Hum Mol Genet. 2001;10(1):1-8.

14. Patrakka J, et al. Congenital nephrotic syndrome (NPHS1): features resulting from different mutations in Finnish patients. Kidney Int. 2000;58(3):972-980.

15. Ruotsalainen V, et al. Role of nephrin in cell junction formation in human nephrogenesis. Am J Pathol. 2000;157(6):1905-1916.

16. Patrakka J, et al. Proteinuria and prenatal diagnosis of congenital nephrosis in fetal carriers of nephrin gene mutations. Lancet. 2002;359(9317):1575-1577.

17. Schneeberger EE, Levey RH, McCluskey RT, Karnovsky MJ. The isoporous substructure of the human glomerular slit diaphragm. Kidney Int. 1975;8(1):48-52.

18. Al-Amoudi A, Díez DC, Betts MJ, Frangakis AS. The molecular architecture of cadherins in native epidermal desmosomes. Nature. 2007;450(7171):832-837.

19. Özkan E, et al. Extracellular architecture of the SYG-1/SYG-2 adhesion complex instructs synaptogenesis. Cell. 2014;156(3):482-494.

20. Sauer F, Vahokoski J, Song YH, Wilmanns M. Molecular basis of the head-to-tail assembly of giant muscle proteins obscurinlike 1 and titin. EMBO Rep. 2010;11(7):534-540.

21. Yoshida Y, et al. Proteomic approach to human kidney glomerulus prepared by laser microdissection from frozen biopsy specimens: exploration of proteome after removal of blood-derived proteins. Proteomics Clin Appl. 2012;6(7-8):412-417.

22. Boerries M, et al. Molecular fingerprinting of the podocyte reveals novel gene and protein regulatory networks. Kidney Int 2013;83(6):1052-1064

23. Boute N, et al. NPHS2, encoding the glomerular protein podocin, is mutated in autosomal recessive steroid-resistant nephrotic syndrome. Nature Genetics. 2000;24(4):349-354.

24. Shih NY, et al. Congenital nephrotic syndrome in mice lacking CD2-associated protein. Science. 1999;286(5438):312-315.

25. Winn MP, et al. A mutation in the TRPC6 cation channel causes familial focal segmental glomerulosclerosis. Science. 2005;308(5729):1801-1804.

26. Reiser J, et al. TRPC6 is a glomerular slit diaphragm-associated channel required for normal renal function. Nat Genet. 2005;37(7):739-744

27. Schnabel E, Anderson JM, Farquhar MG. The tight junction protein ZO-1 is concentrated along slit diaphragms of the glomerular epithelium. J Cell Biol. 1990;111(3):1255-1263.

28. Hamano Y, et al. Determinants of vascular permeability in the kidney glomerulus. J Biol Chem. 2002;277(34):31154-31162.

29. Rantanen M, et al. Nephrin TRAP mice lack slit diaphragms and show fibrotic glomeruli and cystic tubular lesions. $J$ Am Soc Nephrol. 2002;13(6):1586-1594.

30. Rinta-Valkama J, Palmén T, Lassila M, Holthöfer H. Podocyte-associated proteins FAT, alpha-actinin-4 and filtrin are expressed in Langerhans islets of the pancreas. Mol Cell Biochem. 2007;294(1-2):117-125.

31. Puchner EM, et al. Mechanoenzymatics of titin kinase. Proc Natl Acad Sci USA. 2008;105(36):13385-13390.

32. Bertz M, Wilmanns M, Rief M. The titin-telethonin complex is a directed, superstable molecular bond in the muscle Z-disk PNAS. 2009;106(32):13307-133310.

33. Ichimura K, et al. Three-dimensional architecture of podocytes revealed by block-face scanning electron microscopy. Sci Rep. 2015;5:8993.

34. Burghardt T, et al. Advanced electron microscopic techniques provide a deeper insight into the peculiar features of podocytes. Am J Physiol Renal Physiol. 2015;309(12):F1082-F1089.

35. Yaoita E, et al. Avian podocytes, which lack nephrin, use adherens junction proteins at intercellular junctions. J Histochem Cytochem. 2016;64(1):67-76

36. Kelley LA, Sternberg MJ. Protein structure prediction on the Web: a case study using the Phyre server. Nat Protoc. 2009;4(3):363-371. 